\section{Birds of Osmanabad District of Maharashtra, India}

\section{Sujit Narwade ${ }^{1} \&$ M.M. Fartade ${ }^{2}$}

${ }^{1}$ Scientist-in-Charge, ENVIS Centre on Avian Ecology, Bombay Natural History Society, Mumbai, Maharashtra 400001, India

${ }^{2}$ Principal, Shree Sivaji Mahavidhyalaya, Barshi, Solapur District, Maharashtra 413401, India

Email: ${ }^{1}$ sujitsnarwade@gmail.com (corresponding author),

2ssmb barshi@rediffmail.com

Pioneering study of the birds of the Deccan has been done by Davidson \& Wenden (1878). Through an annotated checklist of the birds of western Maharashtra, Prasad (2003) compiled one for Solapur District which is the neighbouring area of our interest. Bharucha \& Gogte (1990) studied the avian profile of a man-modified aquatic ecosystem in the backwaters of the Ujjani Dam while separate lists of birds of Solapur District are available in Rahmani (1989) and Mahabal (1989). Avian ecology of Solapur District, has been well studied, but the Marathwada region of Maharashtra has not yet been explored thoroughly. Through this paper we would like to draw attention towards the least known avifauna of the Osmanabad District.

\section{Study area}

The study area comes under the Marathwada region of Maharashtra (Fig. 1). The forest types are semi arid biotope, open scrublands and southern tropical thorn forest (Champion \& Seth 1968). It

Date of publication (online): 26 February 2011

Date of publication (print): 26 February 2011

ISSN 0974-7907 (online) | 0974-7893 (print)

Editor: Nishith Dharaiya

Manuscript details:

Ms \# 02462

Received 21 May 2010

Final received 28 December 2010

Finally accepted 07 January 2011

Citation: Narwade, S. \& M.M. Fartade (2011). Birds of Osmanabad District of Maharashtra, India. Journal of Threatened Taxa 3(2): 1567-1576.

Copyright: (c) Sujit Narwade \& M.M. Fartade 2011. Creative Commons Attribution 3.0 Unported License. JoTT allows unrestricted use of this article in any medium for non-profit purposes, reproduction and distribution by providing adequate credit to the authors and the source of publication.

\section{OPEN ACCESS | FREE DOWNLOAD (C) (i) आ}

lies on the Deccan plateau at an average of $600 \mathrm{~m}$ above sea level. Osmanabad District comes under

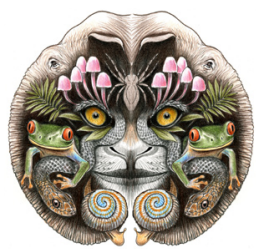
the low rainfall region, with about $600 \mathrm{~mm}$ annual precipitation.

We covered a total of $300 \mathrm{~km}^{2}$ area in three blocks of $10 \times 10 \mathrm{~km}^{2}$ areas at the following selected places.

(i) Yedshi grassland: This includes grassland area spread between northeast of Osmanabad City and Yedshi Town $\left(18^{\circ} 15^{\prime} \mathrm{N} \& 7^{\circ} 59^{\prime} \mathrm{E}\right)$.

(ii) Terna Lake: One of the largest dams of Osmanabad District, located $20 \mathrm{~km}$ northwest of Osmanabad City, near Dhoki Town $\left(18^{0} 19^{\prime} \mathrm{N}\right.$ \& $\left.76^{0} 05^{\prime} \mathrm{E}\right)$.

(iii) Masla: This village is located $38 \mathrm{~km}$ northeast from Solapur City and 20km south from Osmanabad City. Data was collected from a small wetland, agricultural area as well as from near human habitation $\left(17^{\circ} 58^{\prime} \mathrm{N} \& 76^{\circ} 05^{\prime} \mathrm{E}\right)$. We also covered the areas of adjoining villages named Dahivadi, Pangardarwadi, Savargaon, Kemwadi and Malumbra.

\section{Methods}

Bimonthly surveys were carried out in the above mentioned selected areas from March 2007 to July 2008. The areas were surveyed using binoculars and digital cameras for proper bird records. Direct observations and species noting was made by walking on the roads, tracks, grassland and agriculture areas. The observations were carried out at different points around the large wetland. Birds were identified following Ali \& Ripley (1983), Grimmett et al. (2000), and Rasmussen \& Anderton (2005). The list of birds was arranged family wise following Manakadan \& Pittie (2001). Current status of threatened categories was adopted from BirdLife International (2000). Habitat wise species count was carried out in four different habitats such as agriculture, wetland, grassland and near human habitation.

\section{Results}

A total of 165 bird species were recorded during the above mentioned survey. Out of them 109 were resident birds species, 15 were local migratory, 41 species were migratory and eight species were under the threatened category. Fifty families represent the 165 bird species with Muscicapidae comprising small birds at the top, 


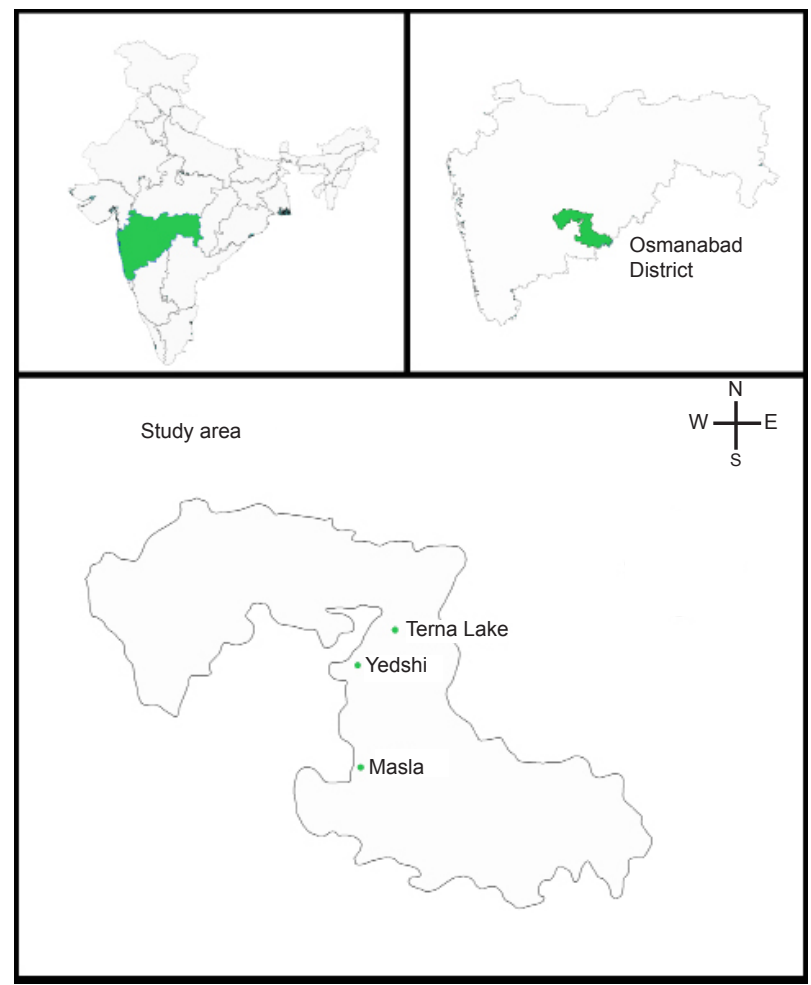

Figure 1. Study area

following Accipitridae (raptors) and Anatidae (mainly ducks), showing a healthy bird diversity in the region (Table 1). Relative commonness of a species has been given as common if species was sighted 7-8 times out of eight visits, fairly common if sighted 4-5 times, uncommon if sighted 2-3 times and rare if sighted 0-2 times out of eight visits. It was observed that out of 165 species, 112 found were common, 11 fairly common, 31 uncommon and 11were rare (Table 2). The detailed information on the threatened birds observed during the surveys has been provided separately in Table 3 .

General observations: Threatened birds such as Pallied Harrier Circus macrourus and European Roller Coracias garrulous (Image 1) were found over all the areas in winter. The Great Indian Bustard Ardeotis nigriceps, an endangered and endemic bird of India and Pakistan was also observed. Three bustards were seen feeding in a farmland area on the boundary of Kemwadi and Savargaon villages in November 2007. This area is just $8-10 \mathrm{~km}$ away from the Great Indian Bustard Sanctuary, Nannaj, Solapur. The sanctuary covers an area of $150 \mathrm{~km}$ from Nannaj towards Ahmednagar District, but part of Osmanabad which is just $10 \mathrm{~km}$ from the core area is still unprotected. A couple of nests and juveniles of ibises and storks found on large trees in Masla Village hinted at a heronry nearby (Images $2 \& 3$ ). We observed a colony of 12 nests of Little Tern Sterna albifrons within a $200 \mathrm{~m}^{2}$ area in the summer of 2007 at Masla Lake. It is important to note that only two confirmed breeding records of Little Tern in Maharashtra are available till

\begin{tabular}{|c|c|c|c|c|c|}
\hline Family & $\begin{array}{c}\text { No. of } \\
\text { species }\end{array}$ & Family & $\begin{array}{c}\text { No. of } \\
\text { species }\end{array}$ & Family & $\begin{array}{c}\text { No. of } \\
\text { species }\end{array}$ \\
\hline Podicipedidae & 1 & Laridae & 3 & Hirundinidae & 4 \\
\hline Phalacrocoracidae & 1 & Pteroclididae & 2 & Campephagidae & 4 \\
\hline Anhingidae & 1 & Columbidae & 3 & Laniidae & 3 \\
\hline Ardeidae & 7 & Psittacidae & 1 & Muscicapidae & 17 \\
\hline Ciconiidae & 3 & Cuculidae & 7 & Paridae & 1 \\
\hline Threskiornithidae & 3 & Tytonidae & 1 & Motacillidae & 7 \\
\hline Anatidae & 10 & Strigidae & 3 & Dicaeidae & 1 \\
\hline Accipitridae & 14 & Caprimulgidae & 1 & Nectariniidae & 2 \\
\hline Phasianidae & 6 & Apodidae & 1 & Zosteropidae & 1 \\
\hline Turnicidae & 3 & Alcedinidae & 2 & Emberizidae & 3 \\
\hline Rallidae & 5 & Meropidae & 1 & Estrildidae & 4 \\
\hline Otididae & 1 & Coraciidae & 2 & Passeridae & 2 \\
\hline Recurvirostridae & 1 & Upupidae & 1 & Sturnidae & 3 \\
\hline Burhinidae & 2 & Bucerotidae & 1 & Oriolidae & 1 \\
\hline Charadriidae & 3 & Capitonidae & 1 & Dicruridae & 1 \\
\hline Scolopacidae & 8 & Picidae & 1 & Corvidae & 3 \\
\hline Glareolidae & 3 & Alaudidae & 5 & & \\
\hline
\end{tabular}




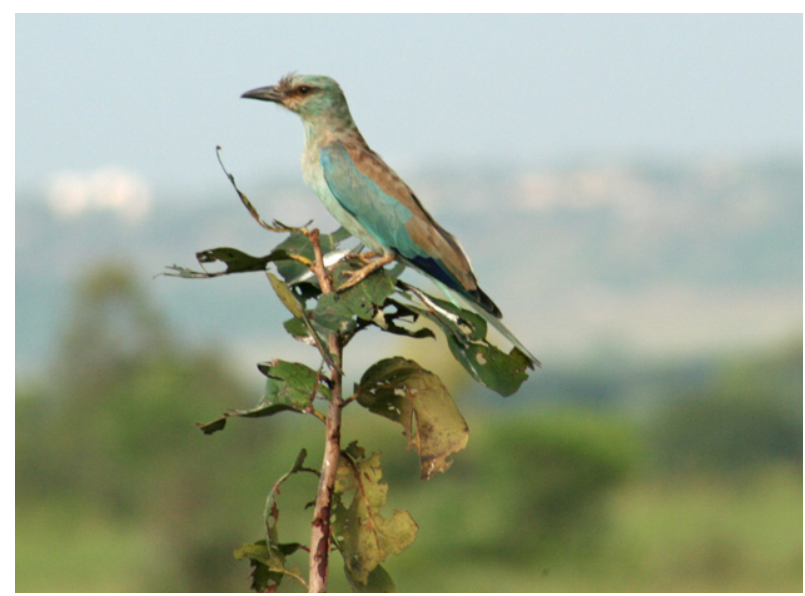

Image 1. European Roller Coracias garrulous

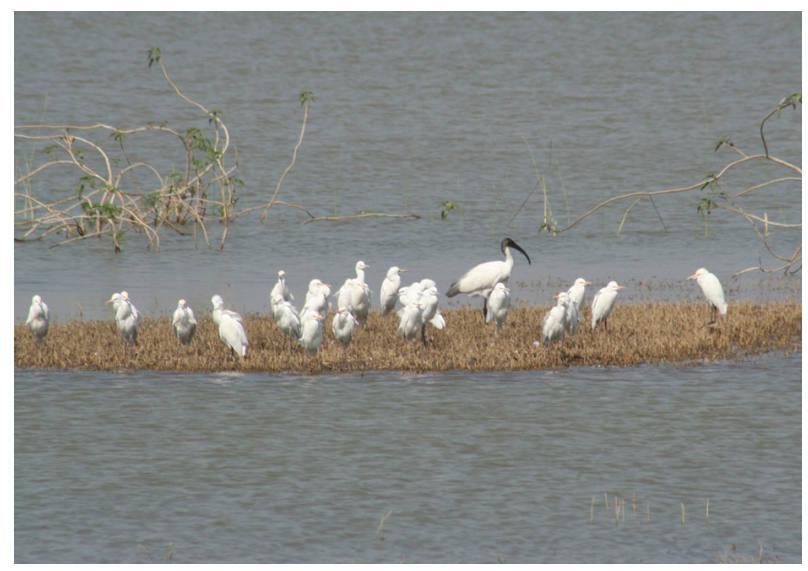

Image 3. Black-headed Ibis Threskiornis aethiopica

date (Ali \& Ripley 1983; Bharucha \& Gogte 1990).

Habitat wise seasonal changes in species count: A pie chart of habitat wise species count was prepared (Fig. 2) which indicates the use of the habitats by the

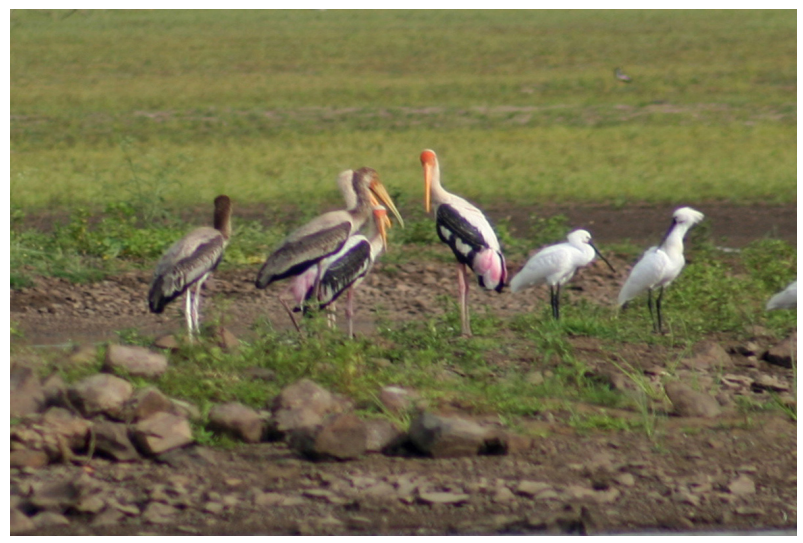

Image 2. Painted Stork with juveniles

birds. The highest number of bird species was found in wetland areas following agricultural and grassland areas. Eleven species were found using all four habitats commonly. The highest number of species i.e. 130 in winter, indicates that the area provides suitable ground for many migratory birds. In summer many birds found in the afternoon, roosting near the edge of the water body to escape from the high temperatures, used to disperse into nearby areas in the evening. Juveniles and subadults of the ground nesting birds observed in winter indicate that the period of late summer and monsoons was the major breeding season. The population of some of the birds such as Blackwinged Stilt Himantopus himantopus was found high in winter, but a couple of birds observed in summer and the monsoons confirmed that some of the birds are residents. Birds such as lapwings and larks were found using wetland habitat extensively for nesting in their breeding season (Narwade et al. in press). In winter,

Table 3. Observation of the threatened birds of Osmanabad District

\begin{tabular}{|c|c|c|c|c|c|}
\hline Species & Season & Area & Habitat & $\begin{array}{l}\text { No. of } \\
\text { birds }\end{array}$ & Activity \\
\hline Great Indian Bustard Ardeotis nigriceps (EN) & $\begin{array}{l}\text { Monsoon } \\
\text { Winter }\end{array}$ & $\begin{array}{l}\text { Devkuruli-Katgaon area } \\
\text { Kemwadi-Savargaon }\end{array}$ & $\begin{array}{l}\mathrm{G} \\
\mathrm{A}\end{array}$ & $\begin{array}{l}1 \\
3\end{array}$ & $\begin{array}{l}\text { Flying } \\
\text { Feeding }\end{array}$ \\
\hline Indian Spotted Eagle Aquila hastate (VU) & Summer & Terna & W & 1 & Soaring \\
\hline Oriental Darter Anhinga melanogaster (NT) & Summer & Terna & W & 1 & Basking \\
\hline Painted Stork Mycteria leucocephala (NT) & $\begin{array}{l}\text { Summer } \\
\text { Monsoon }\end{array}$ & $\begin{array}{l}\text { Masla, Terna } \\
\text { Masla, Terna }\end{array}$ & $\begin{array}{l}\text { W } \\
W\end{array}$ & $\begin{array}{c}45 \\
7\end{array}$ & $\begin{array}{l}\text { Resting } \\
\text { Feeding }\end{array}$ \\
\hline Black-headed Ibis Threskiornis aethiopica (NT) & Monsoon & Terna & W & 4 & Feeding \\
\hline Pallid Harrier Circus macrourus (NT) & Winter & Yedhsi, Masla & $\begin{array}{l}\mathrm{G} \\
\mathrm{G}\end{array}$ & $\begin{array}{c}14 \\
4\end{array}$ & Flying \\
\hline European Roller Coracias garrulous (NT) & Winter & Masla & $\mathrm{G} / \mathrm{A}$ & 7 & Feeding \\
\hline Black-tailed Godwit Limosa limosa (NT) & Winter & Terna & W & 4 & Feeding \\
\hline
\end{tabular}

EN - Endangered; NT - Near Threatened; VU - Vulnerable; A - Agriculture; G - Grassland; W - Wetland. 


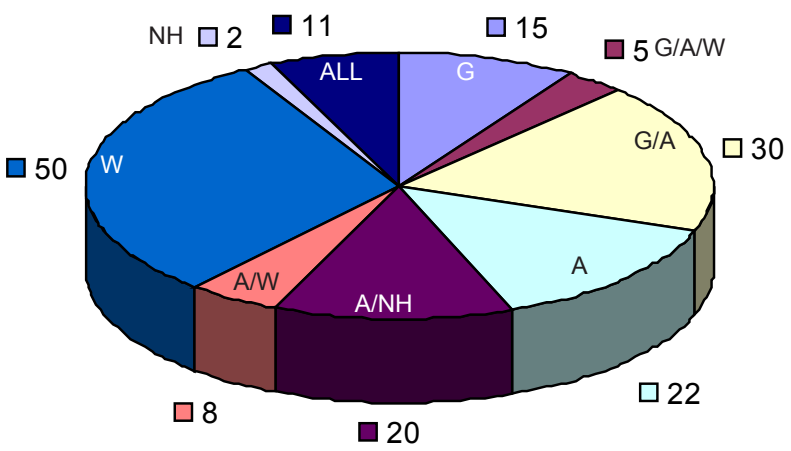

Figure 2. Number of bird species observed in various habitats

A - Agriculture; G - Grassland; W - Wetland; NH - Near Human habitation; / - Sign indicates and.

large flocks of small birds such as buntings, larks, weavers and sparrows were observed in agricultural areas. Seasonal variations observed in the species count at different habitats needs to be studied in detail for the bird conservation.

\section{Discussion}

The major cropping season here is during the monsoon, from July onwards and harvesting is carried out in October-November. Farmers cultivated short term crops such as Urid in monsoon followed by Jowar Sorghum vulgare as one of the major Kharif crops. Harvesting was carried out during JanuaryFebruary after which a large area of cropland becomes fallow due to the high temperatures and scarce water supply. This lean time was used by the people for social interactions such as weddings, which thus resulted in fewer disturbances to the wildlife. But the hunting communities got active in summer because of poor income resources which again put pressure on the wildlife. It was observed that hunters usually hunt for blackbuck, hare, quail and partridge. Rapid developmental activities are continuing to increase the crop yield, as well as, the sources for income. Factors such as use of machines in farming activities, irrigation facilities, use of fertilizers and pesticides are responsible for rapid changes in the cropping patterns throughout the area. Agricultural practices in the study area were found affecting the breeding success of ground nesting birds (Narwade et al. 2010).

Due to the developmental activities the entire habitat of this district has become vulnerable to the upcoming changes. The ambitious lake linking programmes by the Godavari Marathwada Irrigation Development
Corporation (GMIDC) has been launched by the Government of Maharashtra. This may be beneficial to the farmers and wetland birds, but the existing grassland avifauna is under great threat.

The area still provides some potential habitats for the declining population of the threatened birds. It is the need of the hour to monitor these areas systematically in the rapidly changing environment with a focused study on status, distribution and conservation of the avifauna of the region. This can be achieved only through strengthening public participation in the study of status, distribution and conservation of birds of Marathwada region, Maharashtra. Once recorded as common birds of the Deccan, the Lesser Florican (EN), Black-necked Stork (NT), Red-headed Vulture (CR), White-rumped Vulture (CR), Sociable Lapwing (CR), Greater Adjutant, Lesser Adjutant (VU) were not sighted during our surveys. There is a great need to carry out proper and systematic surveys in the large geographical area of the Marathwada region of Maharashtra, India.

\section{REFERENCES}

Ali, S. \& S.D. Ripley (1983). Compact Edition of Handbook of the Birds of India and Pakistan. Bombay Natural History Society and Oxford University Press, Bombay, 737pp.

Bharucha, E.K. \& P.P. Gogte (1990). Avian profile of a manmodified aquatic ecosystem in the backwaters of the Ujjani Dam. Journal of the Bombay Natural History Society 87: 73-90.

BirdLife International (2000). Threatened Birds of the World. Barcelona and Cambridge, U.K. Lynx Edicions and BirdLife International, xii+852pp.

Champion, H.G. \& S.K. Seth (1968). A Revised Survey of the Forest Types of India. Government of India, Delhi, 402pp.

Davidson, C.S. \& C.E. Wenden (1878). A contribution to the avifauna of the Deccan. Stray Feathers 7: 68-95.

Grimmett, R., C. Inskipp \& T. Inskipp (2000). Birds of the Indian Subcontinent. Oxford University Press, New Delhi, $384 \mathrm{pp}$.

Mahabal,A. (1989). Avifauna of Sholapur district, Maharashtra, a semi arid biotope. Records of the Zoological Survey of India 85(4): 589-607.

Manakadan, R. \& A. Pittie (2001). Standardised common and scientific names of the birds of the Indian Subcontinent. Buceros (ENVIS Newsletter) 6(1): i-ix, 1-37.

Narwade, S.S., K.M. Fartade \& M.M. Fartade (2010). Effect of agricultural activities on breeding success of Redwattled Lapwing Vanellus indicus. National Journal of Life Sciences 7(1): 31-34. 
Table 2. List of birds of Osmanabad District, Maharashtra, India

\begin{tabular}{|c|c|c|c|c|c|c|c|}
\hline & Common and scientific names & Habitat & Site name & Activity & Season & Abundance & $\begin{array}{c}\mathrm{R} / \mathrm{LM} / \\
\mathrm{M}\end{array}$ \\
\hline & Podicipedidae & & & & & & \\
\hline \multirow[t]{2}{*}{1} & Little Grebe Podiceps ruficollis & W & Terna & Breeding (chicks) & Monsoon & Fairly common & $\mathrm{R}$ \\
\hline & Phalacrocoracidae & & & & & & \\
\hline \multirow[t]{2}{*}{2} & Little Cormorant Phalacrocorax niger & W & Terna & Drying wings & All & Common & $\mathrm{R}$ \\
\hline & Anhingidae & & & & & & \\
\hline \multirow[t]{2}{*}{3} & Darter Anhinga melanogaster & W & Terna & Swimming & Winter & Rare & LM \\
\hline & Ardeidae & & & & & & \\
\hline 4 & Grey Heron Ardea cinerea & W & Terna, Masla & Feeding & Summer & Fairly common & LM \\
\hline 5 & Indian Pond Heron Ardeola grayii & W & Terna, Masla & Feeding & All & Common & $\mathrm{R}$ \\
\hline 6 & Cattle Egret Bubulcus ibis & W/A & All areas & Feeding & All & Common & $\mathrm{R}$ \\
\hline 7 & Intermediate Egret Egretta intermedia & W & Terna & Roosting & Winter & Uncommon & M \\
\hline 8 & Great Egret Egretta alba & W & Terna & Roosting & Summer & Uncommon & LM \\
\hline 9 & Little Egret Egretta garzetta & W & All areas & Feeding & All & Fairly common & LM \\
\hline \multirow[t]{2}{*}{10} & Purple Heron Ardea purpurea & W & Terna & Feeding & Winter & Rare & M \\
\hline & Ciconiidae & & & & & & \\
\hline 11 & Painted Stork Mycteria leucocephala & W & Terna, Masla & $\begin{array}{l}\text { Roosting along } \\
\text { with Juveniles }\end{array}$ & Summer & Fairly common & $\mathrm{R}$ \\
\hline 12 & Asian Openbill Anastomus oscitans & W & Terna & Feeding & Summer & Uncommon & LM \\
\hline \multirow[t]{2}{*}{13} & Woolly-necked Stork Ciconia episcopus & W/A/G & All areas & $\begin{array}{l}\text { Breeding (Nest, } \\
\text { chick), Feeding }\end{array}$ & All & Common & $\mathrm{R}$ \\
\hline & Threskiornithidae & & & & & & \\
\hline 14 & Black-headed Ibis Threskiornis aethiopica & W/A & Terna & $\begin{array}{l}\text { Feeding with a } \\
\text { Juveniles }\end{array}$ & Summer & Uncommon & LM \\
\hline 15 & Black Ibis Pseudibis papillosa & W/A & Masla & Feeding & Summer & Common & $\mathrm{R}$ \\
\hline \multirow[t]{2}{*}{16} & Eurasian Spoonbill Platalea leucorodia & W & Masla & Breeding & All & Common & $\mathrm{R}$ \\
\hline & Anatidae & & & & & & \\
\hline 17 & Ruddy Shelduck Tadorna ferruginea & W & Terna & Swimming & Winter & Common & M \\
\hline 18 & Northern Pintail Anas acuta & W & Terna & Swimming & Winter & Common & M \\
\hline 19 & Common Teal Anas crecca & W & Terna & Swimming & Winter & Uncommon & M \\
\hline 20 & Spot-billed Duck Anas poecilorhyncha & W & $\begin{array}{l}\text { All wetland } \\
\text { areas }\end{array}$ & $\begin{array}{l}\text { Breeding, } \\
\text { Feeding }\end{array}$ & All & Common & $\mathrm{R}$ \\
\hline 21 & Gadwall Anas strepera & W & Terna & Swimming & Winter & Fairly common & M \\
\hline 22 & Eurasian Wigeon Anas penelope & W & Terna & Swimming & Winter & Fairly common & M \\
\hline 23 & Garganey Anas querquedula & W & Terna & Swimming & Winter & Common & M \\
\hline 24 & Northern Shoveler Anas clypeata & W & Terna & Swimming & Winter & Common & M \\
\hline 25 & Common Pochard Aythya ferina & W & Terna & Swimming & Winter & Uncommon & M \\
\hline \multirow[t]{2}{*}{26} & Comb Duck Sarkidionlis melanotos & W & Terna, Masla & Roosting & Summer & Common & LM \\
\hline & Accipitridae & & & & & & \\
\hline 27 & Black-shouldered Kite Elanus caeruleus & All & All areas & Perching & All & Common & $\mathrm{R}$ \\
\hline 28 & Black Kite Milvus migrans & $\begin{array}{c}\mathrm{G} / \mathrm{W} / \mathrm{A} / \\
\mathrm{NH}\end{array}$ & All areas & $\begin{array}{l}\text { Feeding, } \\
\text { breeding }\end{array}$ & All & Common & $\mathrm{R}$ \\
\hline 29 & Brahminy Kite Haliastur indus & W/A & All areas & Perching & All & Common & $\mathrm{R}$ \\
\hline 30 & Shikra Accipiter badius & $A / G$ & Masla & Perching & All & Common & $\mathrm{R}$ \\
\hline 31 & White-eyed Buzzard Butastur teesa & A/G & Yedshi & Perching & All & Uncommon & $\mathrm{R}$ \\
\hline 32 & Pallid Harrier Circus macrourus & $A / G$ & Masla & Roosting & Winter & Common & M \\
\hline 33 & $\begin{array}{l}\text { Oriental Honey Buzzard Pernis } \\
\text { ptilorhynchus }\end{array}$ & $A / G$ & Yedshi & Perching & All & Uncommon & $\mathrm{R}$ \\
\hline
\end{tabular}




\begin{tabular}{|c|c|c|c|c|c|c|c|}
\hline & Common and scientific names & Habitat & Site name & Activity & Season & Abundance & $\begin{array}{c}\mathrm{R} / \mathrm{LM} / \\
\mathrm{M}\end{array}$ \\
\hline 34 & Montagu's Harrier Circus pygargus & G & Masla & Roosting & Winter & Uncommon & M \\
\hline 35 & Eurasian Marsh Harrier Circus aeruginosus & W/G & Terna & Feeding & Winter & Common & M \\
\hline 36 & Crested Serpent Eagle Spilornis cheela & W & Terna & Perching & Winter & Rare & $\mathrm{R}$ \\
\hline 37 & Short-toed Eagle Circaetus gallicus & G & Yedshi & Perching & Winter & Rare & $\mathrm{R}$ \\
\hline 38 & Red-necked Falcon Falco chicquera & $A / G$ & Masla & Perching & Monsoon & Common & $\mathrm{R}$ \\
\hline 39 & Common Kestrel Falco tinnunculus & G & Masla & Perching & Winter & Rare & LM \\
\hline \multirow[t]{2}{*}{40} & Bonelli's Eagle Hieraaetus fasciatus & G & Masla & Perching & Monsoon & Common & $\mathrm{R}$ \\
\hline & Phasianidae & & & & & & \\
\hline 41 & Painted Francolin Francolinus pictus & $A / G$ & All areas & Calling, Breeding & All & Common & $\mathrm{R}$ \\
\hline 42 & Grey Partridge Francolinus pondicerianus & $\mathrm{A} / \mathrm{G}$ & All areas & Calling, Breeding & All & Common & $\mathrm{R}$ \\
\hline 43 & Rain Quail Coturnix coromandelica & $A / G$ & All areas & Calling & Monsoon & Common & M \\
\hline 44 & Rock Bush Quail Perdicula argoondoh & $A / G$ & Masla & Moving & Monsoon & Uncommon & $\mathrm{R}$ \\
\hline 45 & Jungle Bush Quail Perdicula asiatica & $A / G$ & Masla & Moving & Monsoon & Common & $\mathrm{R}$ \\
\hline \multirow[t]{2}{*}{46} & Indian Peafowl Pavo cristatus & $A / G$ & All areas & Calling, Feeding & All & Common & $\mathrm{R}$ \\
\hline & Turnicidae & & & & & & \\
\hline 47 & Barred Buttonquail Turnix suscitator & $A / G$ & All areas & Calling & All & Common & $\mathrm{R}$ \\
\hline 48 & Yellow-legged Buttonquail Turnix tanki & G & Yedshi & Moving & Monsoon & Rare & $\mathrm{R}$ \\
\hline \multirow[t]{2}{*}{49} & Small Buttonquail Turnix sylvaticus & G & Yedshi & Moving & Monsoon & Rare & $\mathrm{R}$ \\
\hline & Rallidae & & & & & & \\
\hline 50 & $\begin{array}{l}\text { White-breasted Waterhen Amaurornis } \\
\text { phoenicurus }\end{array}$ & W & Masla, Terna & Feeding & All & Common & $\mathrm{R}$ \\
\hline 51 & Purple Swamphen Porphyrio porphyrio & W & Terna & Feeding & Monsoon & Common & $\mathrm{R}$ \\
\hline 52 & Common Moorhen Gallinula chloropus & W & Terna & Feeding & All & Uncommon & $\mathrm{R}$ \\
\hline 53 & Eurasian Coot Fulica atra & W & Terna & Feeding & All & Common & $\mathrm{R}$ \\
\hline \multirow[t]{2}{*}{54} & Brown Crake Porzana akool & $\mathrm{A} / \mathrm{W}$ & Masla & Moving & Monsoon & Rare & $\mathrm{R}$ \\
\hline & Otididae & & & & & & \\
\hline \multirow[t]{2}{*}{55} & Great Indian Bustard Ardeotis nigriceps & $\mathrm{A} / \mathrm{G}$ & $* * *$ & Flying & Monsoon & Uncommon & LM \\
\hline & Recurvirostridae & & & & & & \\
\hline \multirow[t]{2}{*}{56} & Black-winged Stilt Himantopus himantopus & W & Masla, Terna & Feeding & $\begin{array}{l}\text { Summer, } \\
\text { Winter }\end{array}$ & Common & $\mathrm{R}$ \\
\hline & Burhinidae & & & & & & \\
\hline 57 & Eurasian Thick-knee Burhinus oedicnemus & W & Masla & Feeding & Summer & Common & $\mathrm{R}$ \\
\hline \multirow[t]{2}{*}{58} & $\begin{array}{l}\text { Eurasian Stonecurlew Burhinus } \\
\text { oedicnemus }\end{array}$ & $A / G$ & Masla & Feeding & Monsoon & Common & $\mathrm{R}$ \\
\hline & Charadriidae & & & & & & \\
\hline 59 & Red-wattled Lapwing Vanellus indicus & G/W/A & All areas & $\begin{array}{l}\text { Breeding ( } 23 \\
\text { nests) }\end{array}$ & Summer & Common & $\mathrm{R}$ \\
\hline 60 & $\begin{array}{l}\text { Yellow-wattled Lapwing Vanellus } \\
\text { malabaricus }\end{array}$ & $\mathrm{G} / \mathrm{W}$ & All areas & $\begin{array}{l}\text { Breeding (14 } \\
\text { nests) }\end{array}$ & Summer & Common & LM \\
\hline \multirow[t]{2}{*}{61} & Little Ringed Plover Charadrius dubius & W & Masla, Terna & $\begin{array}{l}\text { Breeding (one } \\
\text { nest) }\end{array}$ & Summer & Common & $\mathrm{R}$ \\
\hline & Scolopacidae & & & & & & \\
\hline 62 & Common Redshank Tringa totanus & W & Masla, Terna & Feeding & Winter & Uncommon & M \\
\hline 63 & Spotted Redshank Tringa erythropus & W & Masla, Terna & Feeding & Winter & Common & M \\
\hline 64 & Wood Sandpiper Tringa glareola & W & Masla & Feeding & Winter & Common & M \\
\hline 65 & Common Sandpiper Actitis hypoleucos & W & Masla & Feeding & Winter & Common & $\mathrm{R}$ \\
\hline 66 & $\begin{array}{l}\text { Greater Painted Snipe Rostratula } \\
\text { bengalensis }\end{array}$ & W & Terna & Feeding & Winter & Uncommon & $\mathrm{R}$ \\
\hline 67 & Common Greenshank Tringa nebularia & W & Terna & Feeding & Winter & Uncommon & $\mathrm{R}$ \\
\hline
\end{tabular}




\begin{tabular}{|c|c|c|c|c|c|c|c|}
\hline & Common and scientific names & Habitat & Site name & Activity & Season & Abundance & $\begin{array}{c}\mathrm{R} / \mathrm{LM} / \\
\mathrm{M}\end{array}$ \\
\hline 68 & Marsh Sandpiper Tringa stagnatilis & W & Terna & Feeding & Winter & Rare & M \\
\hline \multirow[t]{2}{*}{69} & Black-tailed Godwit Limosa limosa & W & Terna & Feeding & Winter & Rare & M \\
\hline & Glareolidae & & & & & & \\
\hline 70 & Indian Courser Cursorius coromandelicus & G & Masla & Feeding & Monsoon & Common & LM \\
\hline 71 & Little Stint Calidris minuta & W & Terna & Feeding & Winter & Uncommon & M \\
\hline \multirow[t]{2}{*}{72} & Small Pratincole Glareola lactea & W & Terna & Feeding & Summer & Common & $\mathrm{R}$ \\
\hline & Laridae & & & & & & \\
\hline 73 & Indian River Tern Sterna aurantia & W & Masla & Feeding & Summer & Common & $\mathrm{R}$ \\
\hline 74 & Little Tern Sterna albifrons & W & Masla & $\begin{array}{l}\text { Breeding (8 } \\
\text { nests) }\end{array}$ & Summer & Common & M \\
\hline \multirow[t]{2}{*}{75} & Whiskered Tern Chlidonias hybrida & W & Terna & Roosting & Winter & Fairly Common & M \\
\hline & Pteroclididae & & & & & & \\
\hline 76 & $\begin{array}{l}\text { Chestnut-bellied Sandgrouse Pterocles } \\
\text { exustus }\end{array}$ & G & Masla & Breeding & All & Common & $\mathrm{R}$ \\
\hline \multirow[t]{2}{*}{77} & Painted Sandgrouse Pterocles indicus & G & Yedshi & Breeding & All & Common & $\mathrm{R}$ \\
\hline & Columbidae & & & & & & \\
\hline 78 & Blue Rock Pigeon Columba livia & $\mathrm{A} / \mathrm{NH}$ & Masla & Feeding & All & Common & $\mathrm{R}$ \\
\hline 79 & $\begin{array}{l}\text { Eurasian Collared Dove Streptopelia } \\
\text { decaocto }\end{array}$ & $\mathrm{A} / \mathrm{NH} / \mathrm{G}$ & All areas & Perching & All & Common & $\mathrm{R}$ \\
\hline \multirow[t]{2}{*}{80} & $\begin{array}{l}\text { Little Brown Dove Streptopelia } \\
\text { senegalensis }\end{array}$ & $\mathrm{A} / \mathrm{NH}$ & All areas & Feeding & All & Common & $\mathrm{R}$ \\
\hline & Psittacidae & & & & & & \\
\hline \multirow[t]{2}{*}{81} & Rose-ringed Parakeet Psittacula krameri & A & All areas & Calling & All & Common & $\mathrm{R}$ \\
\hline & Cuculidae & & & & & & \\
\hline 82 & Pied Cuckoo Clamator jacobinus & $\mathrm{A} / \mathrm{NH}$ & Masla & Perching & Monsoon & Uncommon & LM \\
\hline 83 & $\begin{array}{l}\text { Grey-bellied Cuckoo Cacomantis } \\
\text { passerinus }\end{array}$ & A & Terna & Perching & Monsoon & Uncommon & LM \\
\hline 84 & Common Hawk Cuckoo Hierococcyx varius & $\mathrm{A} / \mathrm{NH}$ & Terna & Perching & Monsoon & Common & LM \\
\hline 85 & Indian cuckoo Cuculus micropterus & A & Terna & Perching & Monsoon & Uncommon & LM \\
\hline 86 & Asian Koel Eudynamys scolopacea & $\mathrm{A} / \mathrm{NH}$ & All areas & Calling & All & Common & $\mathrm{R}$ \\
\hline 87 & Southern Coucal Centropus sinensis & A & All areas & Feeding & All & Common & $\mathrm{R}$ \\
\hline \multirow[t]{2}{*}{88} & Sirkeer Malkoha Taccocua leschenaultii & A & Masla & Feeding & Summer & Rare & $\mathrm{R}$ \\
\hline & Tytonidae & & & & & & \\
\hline \multirow[t]{2}{*}{89} & Barn Owl Tyto alba & $\mathrm{NH}$ & All areas & calling & All & Common & $\mathrm{R}$ \\
\hline & Strigidae & & & & & & \\
\hline 90 & Spotted Owlet Athene brama & $\mathrm{A} / \mathrm{W} / \mathrm{NH}$ & Masla & Perching & All & Common & $\mathrm{R}$ \\
\hline 91 & Short-eared Owl Asio flammeus & A & Masla & Perching & Winter & Uncommon & M \\
\hline \multirow[t]{2}{*}{91} & Indian Eagle-owl Bubo bengalensis & W/A & Terna & Perching & All & Common & $\mathrm{R}$ \\
\hline & Caprimulgidae & & & & & & \\
\hline \multirow[t]{2}{*}{93} & Indian Nightjar Caprimulgus asiaticus & $A / G$ & $\begin{array}{l}\text { Yedshi, } \\
\text { Terna }\end{array}$ & Calling & Winter & Common & $\mathrm{R}$ \\
\hline & Apodidae & & & & & & \\
\hline \multirow[t]{2}{*}{94} & Little Swift Apus affinis & $\mathrm{A} / \mathrm{NH}$ & Yedshi & Flying & All & Common & $\mathrm{R}$ \\
\hline & Alcedinidae & & & & & & \\
\hline 95 & Pied Kingfisher Ceryle rudis & W & Masla & Feeding & All & Common & $\mathrm{R}$ \\
\hline 96 & $\begin{array}{l}\text { White-breasted Kingfisher Halcyon } \\
\text { smyrnensis }\end{array}$ & A/W & All areas & Perching & All & Common & $\mathrm{R}$ \\
\hline
\end{tabular}




\begin{tabular}{|c|c|c|c|c|c|c|c|}
\hline & Common and scientific names & Habitat & Site name & Activity & Season & Abundance & $\begin{array}{c}\mathrm{R} / \mathrm{LM} / \\
\mathrm{M}\end{array}$ \\
\hline & Meropidae & & & & & & \\
\hline \multirow[t]{2}{*}{97} & Green Bee-eater Merops orientalis & $A / G$ & All areas & Perching, calling & Winter & Common & $\mathrm{R}$ \\
\hline & Coraciidae & & & & & & \\
\hline 98 & European Roller Coracias garrulous & $A / G$ & Masla & Perching, calling & Winter & Common & M \\
\hline \multirow[t]{2}{*}{99} & Indian Roller Coracias benghalensis & $A / G$ & Masla & Perching, calling & All & Common & $\mathrm{R}$ \\
\hline & Upupidae & & & & & & \\
\hline \multirow[t]{2}{*}{100} & Common Hoopoe Upupa epops & $A / G$ & All areas & Perching, calling & Winter & Common & M \\
\hline & Bucerotidae & & & & & & \\
\hline \multirow[t]{2}{*}{101} & Indian Grey Hornbill Tockus birostris & A & Masla & Perching, calling & All & Common & $\mathrm{R}$ \\
\hline & Capitonidae & & & & & & \\
\hline \multirow[t]{2}{*}{102} & $\begin{array}{l}\text { Coppersmith Barbet Megalaima } \\
\text { haemacephala }\end{array}$ & $A$ & Masla & Perching & All & Common & $\mathrm{R}$ \\
\hline & Picidae & & & & & & \\
\hline \multirow[t]{2}{*}{103} & $\begin{array}{l}\text { Yellow-crowned Woodpecker Dendrocopos } \\
\text { mahrattensis }\end{array}$ & $A / G$ & Yedshi & Perching & All & Common & $\mathrm{R}$ \\
\hline & Alaudidae & & & & & & \\
\hline 104 & Indian Bush Lark Mirafra erythroptera & $A / G$ & All areas & Perching, calling & All & Common & $\mathrm{R}$ \\
\hline 105 & $\begin{array}{l}\text { Ashy-crowned Sparrow Lark Eremopterix } \\
\text { grisea }\end{array}$ & G & All areas & Calling, Breeding & All & Common & $\mathrm{R}$ \\
\hline 106 & $\begin{array}{l}\text { Rufous-tailed Lark Ammomanes } \\
\text { phoenicurus }\end{array}$ & G & All areas & Calling, Breeding & All & Common & $\mathrm{R}$ \\
\hline 107 & Sykes's Lark Galerida deva & G & All areas & Calling, Breeding & All & Common & $\mathrm{R}$ \\
\hline \multirow[t]{2}{*}{108} & Eastern Skylark Alauda gulgula & G & Terna & sighting & All & Uncommon & $\mathrm{R}$ \\
\hline & Hirundinidae & & & & & & \\
\hline 109 & Barn Swallow Hirundo rustica & $\mathrm{A} / \mathrm{NH}$ & Masla & Near water & Winter & Common & M \\
\hline 110 & Wire-tailed Swallow Hirundo smithii & $\mathrm{A} / \mathrm{W}$ & Terna & Flying & Winter & Fairly common & $\mathrm{R}$ \\
\hline 111 & Streak-throated Swallow Hirundo fluvicola & A & Masla, Terna & Flying & Winter & Fairly common & $\mathrm{R}$ \\
\hline \multirow[t]{2}{*}{112} & Red-rumped Swallow Hirundo daurica & $A / G$ & Masla & Perching & All & Common & $\mathrm{R}$ \\
\hline & Campephagidae & & & & & & \\
\hline 113 & $\begin{array}{l}\text { Common Woodshrike Tephrodornis } \\
\text { pondicerianus }\end{array}$ & $A$ & Terna & Perching & Winter & Uncommon & $\mathrm{R}$ \\
\hline 114 & Common Iora Aegithina tiphia & A & Masla & Calling & All & Common & $\mathrm{R}$ \\
\hline 115 & Red-vented Bulbul Pycnonotus cafer & $\begin{array}{c}\mathrm{A} / \mathrm{W} / \mathrm{G} / \\
\mathrm{NH}\end{array}$ & Masla, Terna & Perching & All & Common & $\mathrm{R}$ \\
\hline \multirow[t]{2}{*}{116} & Red-whiskered Bulbul Pycnonotus jocosus & $\mathrm{A} / \mathrm{W}$ & Terna & Perching & All & Uncommon & $\mathrm{R}$ \\
\hline & Laniidae & & & & & & \\
\hline 117 & Southern Grey Shrike Lanius meridionalis & $\mathrm{A} / \mathrm{G}$ & Masla & Perching & All & Common & $\mathrm{R}$ \\
\hline 118 & Bay-backed Shrike Lanius vittatus & $A / G$ & Masla & Perching & All & Uncommon & $\mathrm{R}$ \\
\hline \multirow[t]{2}{*}{119} & Long-tailed Shrike Lanius schach & A/G & Masla & Calling & Winter & Common & $\mathrm{R}$ \\
\hline & Muscicapidae & & & & & & \\
\hline 120 & Oriental Magpie-Robin Copsychus saularis & W/NH & Terna & Feeding & All & Common & $\mathrm{R}$ \\
\hline 121 & $\begin{array}{l}\text { Black Redstart Phoenicurus ochruros } \\
\text { rufiventris }\end{array}$ & $A$ & Terna & Bathing & Winter & Common & M \\
\hline 122 & Common Stonechat Saxicola torquata & A & Masla, Terna & Perching & Winter & Common & M \\
\hline 123 & Pied Bushchat Saxicola caprata & A & Masla, Terna & Perching & All & Common & $\mathrm{R}$ \\
\hline 124 & Indian Robin Saxicoloides fulicata & $\mathrm{A} / \mathrm{G} / \mathrm{NH}$ & Masla, Terna & Breeding & All & Common & $\mathrm{R}$ \\
\hline 125 & Bluethroat Luscinia s. svecica & A/W/NH & Masla & Perching & Winter & Common & M \\
\hline 126 & Small Minivet Pericrocotus cinnamomeus & $\mathrm{A} / \mathrm{NH}$ & Masla & Feeding & All & Common & $\mathrm{R}$ \\
\hline
\end{tabular}




\begin{tabular}{|c|c|c|c|c|c|c|c|}
\hline & Common and scientific names & Habitat & Site name & Activity & Season & Abundance & $\begin{array}{c}\mathrm{R} / \mathrm{LM} / \\
\mathrm{M}\end{array}$ \\
\hline 127 & Large Grey Babbler Turdoides malcolmi & $\mathrm{A} / \mathrm{NH}$ & All areas & Calling, breeding & All & Common & $\mathrm{R}$ \\
\hline 128 & Yellow-eyed Babbler Chrysoma sinense & A & Terna, Masla & Feeding & All & Common & $\mathrm{R}$ \\
\hline 129 & Zitting Cisticola Cisticola juncidis & G & Masla & Perching & Winter & Fairly Common & $\mathrm{R}$ \\
\hline 130 & Blue Rockthrush Monticola solitarius & G & Masla & Perching & Winter & Uncommon & M \\
\hline 131 & Plain Prinia Prinia subflava & $\mathrm{A} / \mathrm{NH}$ & Masla & Calling & All & Common & $\mathrm{R}$ \\
\hline 132 & Ashy Prinia Prinia socialis & $\mathrm{A} / \mathrm{NH}$ & Masla & Breeding & All & Common & $\mathrm{R}$ \\
\hline 133 & Grey-breasted Prinia Prinia hodgsonii & $\mathrm{A} / \mathrm{NH}$ & Masla & Calling & All & Common & $\mathrm{R}$ \\
\hline 134 & Jungle Prinia Prinia sylvatica & A & Masla & On ground & All & Uncommon & $\mathrm{R}$ \\
\hline 135 & Common Tailorbird Orthotomus sutorius & $\mathrm{A} / \mathrm{NH}$ & Masla & Calling & All & Common & $\mathrm{R}$ \\
\hline \multirow[t]{2}{*}{136} & Lesser Whitethroat Sylvia curruca & A & Terna & Water Bath & Winter & Uncommon & M \\
\hline & Paridae & & & & & & \\
\hline \multirow[t]{2}{*}{137} & Great Tit Parus major & $\mathrm{A} / \mathrm{NH}$ & Masla & Feeding & Winter & Common & $\mathrm{R}$ \\
\hline & Motacillidae & & & & & & \\
\hline 138 & Yellow-headed Wagtail Motacilla citreola & W & Terna & Feeding & Winter & Common & M \\
\hline 139 & Yellow Wagtail Motacilla flava & $\mathrm{A} / \mathrm{W}$ & Terna & Feeding & Winter & Common & M \\
\hline 140 & Grey Wagtail Motacilla cinerea & W & Terna & Feeding & Winter & Uncommon & M \\
\hline 141 & White Wagtail Motacilla alba & W & Terna & Feeding & Winter & Common & $M$ \\
\hline 143 & $\begin{array}{l}\text { White-browed Wagtail Motacilla } \\
\text { maderaspatensis }\end{array}$ & W & Masla, Terna & Feeding & All & Common & $\mathrm{R}$ \\
\hline 143 & Tree Pipit Anthus trivialis & W & Terna & Perching & Winter & Common & M \\
\hline \multirow[t]{2}{*}{144} & Paddyfield Pipit Anthus rufulus & W & Masla, Terna & Feeding & All & Common & $\mathrm{R}$ \\
\hline & Dicaeidae & & & & & & \\
\hline \multirow[t]{2}{*}{145} & $\begin{array}{l}\text { Pale-billed Flowerpecker Dicaeum } \\
\text { erythrorhynchos }\end{array}$ & A & Masla & Perching & All & Uncommon & $\mathrm{R}$ \\
\hline & Nectariniidae & & & & & & \\
\hline 146 & Purple Sunbird Nectarinia asiatica & A & Masla, Terna & Feeding & All & Common & $\mathrm{R}$ \\
\hline \multirow[t]{2}{*}{147} & $\begin{array}{l}\text { Purple-rumped Sunbird Nectarinia } \\
\text { zeylonica }\end{array}$ & A & Masla, Terna & Feeding & All & Common & $\mathrm{R}$ \\
\hline & Zosteropidae & & & & & & \\
\hline \multirow[t]{2}{*}{148} & Oriental White-eye Zosterops palbebrosa & A & Masla, Terna & Feeding & Winter & Uncommon & $\mathrm{R}$ \\
\hline & Emberizidae & & & & & & \\
\hline 149 & Red-headed Bunting Emberiza bruniceps & $A / G$ & Masla & Perching & Winter & Uncommon & M \\
\hline 150 & Grey-necked Bunting Emberiza buchanani & $\mathrm{A} / \mathrm{G}$ & Masla & Feeding & Winter & Common & M \\
\hline \multirow[t]{2}{*}{151} & $\begin{array}{l}\text { Black-headed Bunting Emberiza } \\
\text { melanocephala }\end{array}$ & $A / G$ & Terna & Perching & Winter & Common & M \\
\hline & Estrildidae & & & & & & \\
\hline 152 & Indian Silverbill Lonchura malabarica & $\mathrm{A} / \mathrm{G}$ & Masla, Terna & Feeding & Winter & Common & $\mathrm{R}$ \\
\hline 153 & Red Avadavat Amandava amandva & A & Masla, Terna & Feeding & All & Common & $\mathrm{R}$ \\
\hline 154 & Black-headed Munia Lonchura malacca & A & Masla, Terna & Feeding & Winter & Common & $\mathrm{R}$ \\
\hline \multirow[t]{2}{*}{155} & Scaly-breasted Munia Lonchura punctulata & A & Masla, Terna & Feeding & All & Common & $\mathrm{R}$ \\
\hline & Passeridae & & & & & & \\
\hline 156 & House Sparrow Passer domesticus & $\mathrm{A} / \mathrm{NH}$ & All areas & Breeding & All & Common & $\mathrm{R}$ \\
\hline \multirow[t]{2}{*}{157} & Baya Weaver Ploceus philippinus & $\mathrm{A} / \mathrm{W}$ & All areas & Breeding & Monsoon & Common & $\mathrm{R}$ \\
\hline & Sturnidae & & & & & & \\
\hline 158 & Rosy Starling Sturnus roseus & $\mathrm{A} / \mathrm{G}$ & Masla & Feeding & Winter & Common & $\mathrm{M}$ \\
\hline 159 & Brahminy Myna Sturnus pagodarum & $\mathrm{A} / \mathrm{NH}$ & Masla, Terna & Feeding & All & Common & $\mathrm{R}$ \\
\hline 160 & Common Myna Acridotheres tristis & $\mathrm{A} / \mathrm{NH}$ & All areas & Feeding & All & Common & $\mathrm{R}$ \\
\hline
\end{tabular}




\begin{tabular}{|c|c|c|c|c|c|c|c|}
\hline & Common and scientific names & Habitat & Site name & Activity & Season & Abundance & $\begin{array}{c}\mathrm{R} / \mathrm{LM} / \\
\mathrm{M}\end{array}$ \\
\hline & Oriolidae & & & & & & \\
\hline \multirow[t]{2}{*}{161} & Eurasian Golden Oriole Oriolus oriolus & $\mathrm{A} / \mathrm{NH}$ & Terna & Perching & Winter & Fairly Common & $\mathrm{R}$ \\
\hline & Dicruridae & & & & & & \\
\hline \multirow[t]{2}{*}{162} & Black Drongo Dicrurus adsimilis & $A / G$ & Masla & Feeding & Summer & Common & $\mathrm{R}$ \\
\hline & Corvidae & & & & & & \\
\hline 163 & House Crow Corvus splendens & $\mathrm{NH}$ & Terna, Masla & Feeding & All & Common & $\mathrm{R}$ \\
\hline 164 & Long-billed Crow Corvus macrorhynchos & $\mathrm{A} / \mathrm{NH}$ & Terna & Calling & All & Common & $\mathrm{R}$ \\
\hline 165 & Rufous Treepie Dendrocitta vagabunda & A & Terna & Calling & All & Common & $\mathrm{R}$ \\
\hline
\end{tabular}

A - Agriculture; G - Grassland; W - Wetland; NH - Near human habitation

Note - Breeding activity includes records such as display by male birds, nest, hatchlings, chicks and juveniles.

Narwade, S.S., K.M. Fartade \& M.M. Fartade (in press). Nesting ecology of Red-wattled Lapwing in agricultural landscape. Accepted by Life Science Bulletin.

Prasad, A. (2003). Annotated checklist of Birds of Western Maharashtra. Buceros 8(2\&3): 174
Rahmani, A.R. (1989). The Great Indian Bustard. Final report. Bombay, BNHS, 234pp.

Rassmusen, P.C. \& J.C. Anderton (2005). Birds of South Asia - The Ripley Guide. Vols.1: Field Guide. Smithsonian Institution and Lynx Edicions, Washington, D.C. \& Barcelona, 378pp. 